\title{
The Protective Effect of Naringenin on Airway Remodeling after Mycoplasma Pneumoniae Infection by Inhibiting Autophagy- Mediated Lung Inflammation and Fibrosis
}

\author{
Yan Lin $(\mathbb{D}$, Dan Tan, Qianna Kan $(\mathbb{D}$, Zhen Xiao $\mathbb{D}$, and Zhiyan Jiang $\mathbb{D}$ \\ Department of Pediatrics, Longhua Hospital, Shanghai University of Traditional Chinese Medicine, 725 South WanPing Road, \\ Shanghai 200032, China \\ Correspondence should be addressed to Zhen Xiao; xiaozhen61@126.com and Zhiyan Jiang; jzycm@sohu.com
}

Received 15 September 2017; Revised 24 November 2017; Accepted 24 December 2017; Published 4 April 2018

Academic Editor: Alex Kleinjan

Copyright ( 2018 Yan Lin et al. This is an open access article distributed under the Creative Commons Attribution License, which permits unrestricted use, distribution, and reproduction in any medium, provided the original work is properly cited.

\begin{abstract}
Our previous study has shown that Chinese medicine, Qingfei Tongluo formula (QTF), has a significantly therapeutic effect to Mycoplasma pneumoniae (MP) pneumonia (MPP). The aim of this study was to investigate the therapeutic effect and mechanism of naringenin (NRG) on MPP which was an important component of QTF. Here, we studied 124 children with or without MPP and compared inflammatory cytokines and fibrinogen-related protein expression with enzyme-linked immunosorbent assay. We also employed a BALB/c mouse model of MPP and divided the mice into three groups: ctrl (normal control mice), MPP (MP-infected mice), and MPP + NRG (MP-infected mice treated with NRG). BEAS-2B cells were used to confirm the relationship between autophagy, inflammation, and fibrosis. The results show proinflammatory cytokines (interleukin- [IL-] 6, IL-1 $\beta$, and tumor necrosis factor- $\alpha$ ), and transforming growth factor beta (TGF- $\beta$ ) expression was significantly increased after MP infection from both clinical and animal experiment. In vivo experimental confirmation showed that NRG treatment decreased MPP-induced lung injury in mice by inhibiting autophagy-mediated inflammatory cytokine expression and pulmonary fibrosis. In vitro experiments confirmed it. These results indicate that NRG treatment suppressed the inflammatory response and pulmonary fibrosis by inhibition of autophagy after MP infection.
\end{abstract}

\section{Introduction}

Mycoplasma pneumoniae (MP) is a common cause of community-acquired pneumonia, mainly in children and young adults, and is well known to cause a wide variety of respiratory and extrapulmonary diseases $[1,2]$. Increasing evidence has confirmed that the symptoms of pneumonia caused by MP are correlated with the induction of proinflammatory cytokine expression and pulmonary fibrosis [3-5]. MP infection can lead to proinflammatory cytokine, tumor necrosis factor- $\alpha$ (TNF- $\alpha$ ), and chemokines, such as interleukin- (IL-) 6, promoting the recruitment of various leukocytes, primarily neutrophils, to the site of infection, eventually leading to lung injury and pulmonary fibrosis.

Autophagy, a conserved eukaryotic stress-response pathway in which cells sequester damaged or surplus proteins and organelles in double-membrane vesicles and deliver them to lysosomes for degradation, also plays a seminal role in antimicrobial host defense. Classic triggers of autophagy include nutrient deprivation and inhibitors of the metabolic regulator mTOR, such as rapamycin [6]. Autophagy is also strongly induced by bacteria, viruses, and fungal organisms that ultimately are enclosed within autophagosomes and digested within the autophagolysosomes $[7,8]$. Autophagy has been implicated in MP infection in cultured macrophages in vitro $[3,9]$. But the relationship between MP-induced autophagy and inflammatory responses remains unknown.

Our previous study found that Chinese medicine, Qingfei Tongluo formula (QTF), has been used for the clinical treatment of Mycoplasma pneumoniae pneumonia (MPP) resulting in a significantly therapeutic effect. Increasing evidence has shown that naringenin (NRG), an important component of QTF, has a regulatory function to fibroblasts and the inflammatory response. Hernandez-Aquino et al. have found 
that NRG can prevent experimental liver fibrosis by blocking TGF- $\beta$ signaling [10]. Hua et al. found that NRG pretreatment inhibits neuroapoptosis and ameliorates cognitive impairment in rats exposed to isoflurane anesthesia by regulating the PI3/Akt/PTEN signaling pathway and suppressing NF- $\kappa$ B-mediated inflammation [11]. NRG also inhibits stress-induced autophagy [12] and has a protective effect on oxidative stress-induced lung damage [13]. This suggests that NRG can inhibit MP-induced inflammatory responses and pulmonary fibrosis by suppressed autophagy.

In this study, we hypothesized that NRG might reduce the inflammatory response, halt the progression of pulmonary fibrosis, and contribute to the improvement of lung function in a murine model of MPP by inhibiting autophagy. For this purpose, we have investigated the mechanisms of action of NRG and its potential efficacy in the treatment of MPP.

\section{Materials and Methods}

2.1. Clinical Specimen Collection and Ethics Statement. Peripheral blood samples were obtained from 60 patients with MPP and from 64 healthy volunteers (Table 1). A standard format elaborated by the principal investigator with clinical features was included for age, sex, and relative inflammatory factors and fibrinogen-related protein. Permission to use the samples for research purposes was obtained and approved by the Ethics Committee of the Longhua Hospital of China, and a written consent was obtained from all patients.

2.2. MP Culture. MP strain ATCC15531 (American Type Culture Collection, Rockville, MD, USA) was cultured in modified Hayflick medium (GZBIOTEST Co. Ltd., Guangdong, China) containing PPLO broth, horse serum, and $25 \%$ yeast extract, which was added along with penicillin $\mathrm{G}$ $(1,000 \mathrm{U} / \mathrm{ml})$, thallium acetate $(0.025 \%)$, glucose $(0.5 \%)$, and phenol red $(0.002 \%)$ at $\mathrm{pH}$ 7.6. MP was cultured at $37^{\circ} \mathrm{C}$ in $5 \% \mathrm{CO}_{2}$ for 7 days.

2.3. BEAS-2B Cell Culture and Viability. The human lung epithelial BEAS-2B cell line (purchased from Procell Life Science Co. Ltd., Wuhan, China) which stably expressing GFP-LC3 [14] was cultured at $37^{\circ} \mathrm{C}$ in a humidified atmosphere with $5 \% \mathrm{CO}_{2}$ in Dulbecco's modified Eagle's medium/F12 medium supplemented with $10 \%$ fetal bovine serum, $100 \mathrm{U} / \mathrm{ml}$ penicillin, and $100 \mathrm{ng} / \mathrm{ml}$ streptomycin (Invitrogen, Tokyo, Japan). To identify the effect of NRG on MP-induced airway fibrosis and inflammation, BEAS2B cells were treated with NRG $(100 \mu \mathrm{M}$, [Figure 1(a)]) or 3-methyladenine (3-Ma, $4 \mathrm{mM}$ ) (purchased from SigmaAldrich [St. Louis, MO, USA]) for $2 \mathrm{~h}$ prior stimulated with $1 \times 10^{6} \mathrm{cfu}$ (colony-forming units) MP for up to $24 \mathrm{~h}$. The control group was treated with normal saline. Then BEAS2B cells were collected for further study.

2.4. Establishment of the MP Infection Model. Four-week-old $\mathrm{BALB} / \mathrm{c}$ mice (mean body weight, $15 \pm 1 \mathrm{~g}$ ) were purchased from Shanghai Sippr Bk Laboratory Animals Co. Ltd. (Shanghai, China) and housed in a specific-pathogen-free
TABLE 1: Characteristics of subjects in the study.

\begin{tabular}{|c|c|c|}
\hline $\begin{array}{l}\text { Demographic } \\
\text { characteristics }\end{array}$ & $\begin{array}{c}\text { Non-Mycoplasma } \\
\text { pneumoniae } \\
(n=64)(\%)\end{array}$ & $\begin{array}{l}\text { Mycoplasma } \\
\text { pneumoniae } \\
(n=60)(\%)\end{array}$ \\
\hline \multicolumn{3}{|l|}{ Sex } \\
\hline Female & $34(53 \%)$ & $38(63 \%)$ \\
\hline Male & $30(47 \%)$ & $22(37 \%)$ \\
\hline \multicolumn{3}{|l|}{ Age } \\
\hline $1-3$ years & $5(7.8 \%)$ & $4(6.7 \%)$ \\
\hline $3-7$ years & $41(64.1 \%)$ & $32(53.3 \%)$ \\
\hline $7-14$ years & $18(28.1 \%)$ & $24(40 \%)$ \\
\hline Average age & $6.51 \pm 2.85$ & $7.01 \pm 2.80$ \\
\hline \multicolumn{3}{|l|}{ Whole blood cell analysis } \\
\hline White blood cell $\left(\times 10^{9}\right)$ & $10.82 \pm 2.38$ & $11.23 \pm 3.24^{*}$ \\
\hline Neutrophils (\%) & $28.79 \pm 13.24$ & $37.38 \pm 12.68^{* *}$ \\
\hline Lymphocytes (\%) & $61.28 \pm 14.26$ & $57.36 \pm 16.38$ \\
\hline Blood platelet $\left(\times 10^{9}\right)$ & $375.9 \pm 132.7$ & $399.8 \pm 142.8$ \\
\hline \multicolumn{3}{|c|}{ Expression of mammary immune-associated factors } \\
\hline $\mathrm{IL}-1 \beta(\mathrm{pg} / \mathrm{ml})$ & $23.67 \pm 6.35$ & $67 \pm 11.33^{* * *}$ \\
\hline IL-6 (pg/ml) & $46.58 \pm 8.67$ & $95 \pm 16.23^{* * *}$ \\
\hline TNF- $\alpha(\mathrm{pg} / \mathrm{ml})$ & $56.34 \pm 8.33$ & $128.98 \pm 14.27^{* * *}$ \\
\hline TGF- $\beta(\mathrm{pg} / \mathrm{ml})$ & $16.87 \pm 5.34$ & $32.45 \pm 8.62^{* * *}$ \\
\hline
\end{tabular}

IL-17: interleukin- (IL-) 17; IL-1 $\beta$ : interleukin- (IL-) $1 \beta$; IL-6: interleukin(IL-) 6; TNF- $\alpha$ : tumor necrosis factor- $\alpha$; TGF- $\beta$ : transforming growth factor- $\beta$. Data are expressed as mean \pm SD with Student's $t$-test analysis. ${ }^{*} P<0.05,{ }^{* *} P<0.01,{ }^{* * *} P<0.001$ against the control values.

environment with ad libitum access to food and water. After anesthesia, all mice were divided randomly into three groups: ctrl (normal control mice), MPP (MPP-infected mice), and $\mathrm{MPP}+\mathrm{NRG}$ (MP-infected mice treated with NRG). On day 1 , mice in the ctrl and MPP groups were treated with normal saline by intragastric administration, whereas mice in the $\mathrm{MPP}+\mathrm{NRG}$ group were treated with NRG $\left(100 \mathrm{mg} \mathrm{kg}^{-1}\right)$ by intragastric administration. Mice in all groups were subjected to anesthesia with $0.5 \%$ pentobarbital sodium at a concentration of $0.25 \mathrm{ml} / 20 \mathrm{~g}$. Then mice in the MPP and MPP + NRG groups were inoculated intranasally with $50 \mu \mathrm{l}$ of $\mathrm{MP}$ at $\sim 1 \times 10^{8} \mathrm{cfu}$ as reported in a previous study [15]. A $50 \mu \mathrm{l}$ inoculation of saline was similarly given to the mice in the control groups. On day 2, the foregoing steps were repeated. On days 3 to 7, mice in the MPP + NRG group were treated with only NRG. Mice in the MPP group or ctrl group were treated with $0.25 \mathrm{ml}$ of normal saline by intragastric administration repeated for three weeks.

After 1 month of treatment, the mice were intraperitoneally anesthetized with $0.05 \mathrm{ml}$ of $10 \%$ chloral hydrate and fixed in the supine position before sample collection. The neck and chest were sterilized with conventional iodine and alcohol, and then the chest was opened with sterile equipment (soaked in $0.1 \%$ diethylpyrocarbonate for $24 \mathrm{~h}$ and then autoclaved). The entire lung was collected and stored in liquid nitrogen. Serum was isolated from peripheral blood. The animal study protocol was approved by the Ethics Committee of Longhua Hospital. 
<smiles>O=C1CC(c2ccc(O)cc2)Oc2cc(O)cc(O)c21</smiles>

(a)

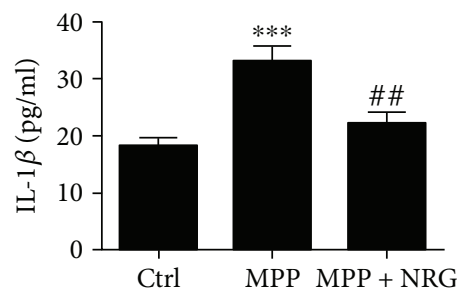

(c)

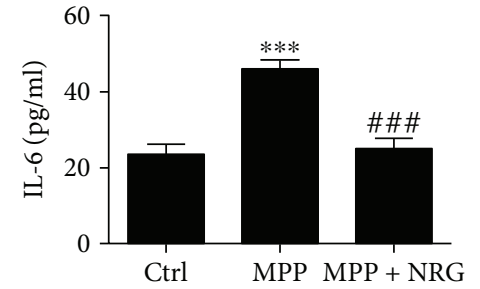

(b)

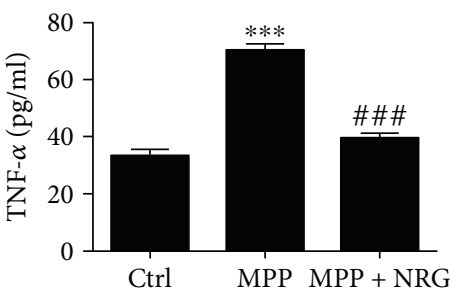

(d)

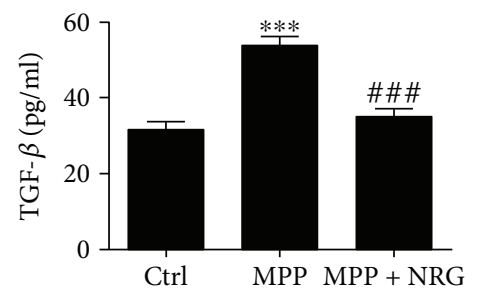

(e)
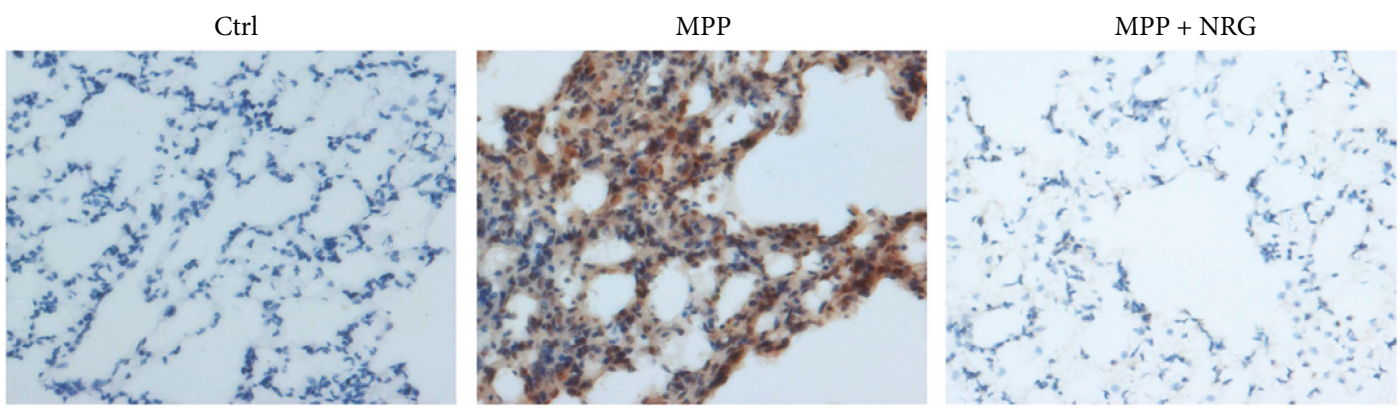

(f)

Ctrl

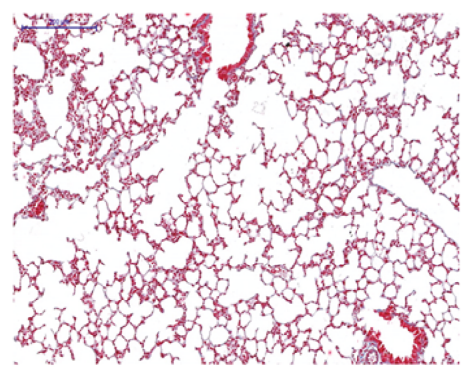

MPP

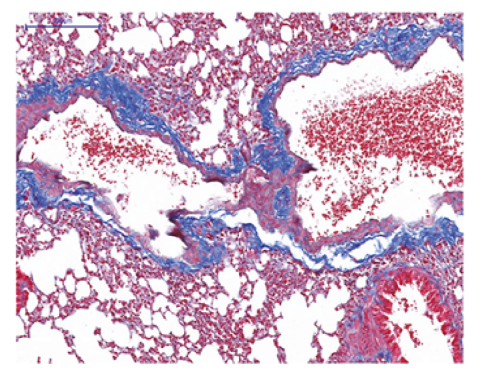

(g)
$\mathrm{MPP}+\mathrm{NRG}$

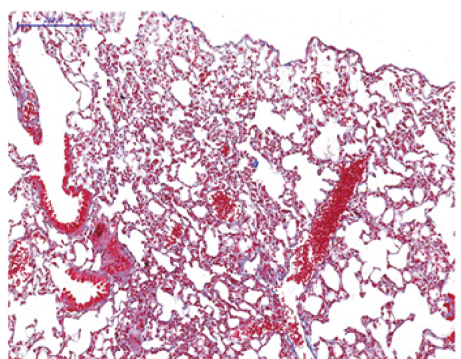

FiguRe 1: NRG treatment decreased $M$. pneumonia-induced lung injury in mice by inhibiting inflammatory cytokine expression and pulmonary fibrosis. (a) Chemical structure of NRG. (b-d) Inflammatory cytokines IL-6 (b), IL-1 $\beta$ (c), TNF- $\alpha$ (d), and fibrosis factor TGF- $\beta$ (e) concentrations in mice serum were measured with ELISA. Data are presented as the mean \pm SD with Tukey-Kramer multiple comparison test analysis. $n=5$. ${ }^{* * *} P<0.001$ versus ctrl group; ${ }^{\#} P<0.01$, \#\# $P<0.001$ versus MPP group. (f and g) Immunohistochemistry shows the presence of apoptosis (TUNEL staining) (f) and fibrosis (Masson trichrome staining) ( $\mathrm{g}$ ) in lung tissues. The results show that NRG treatment significantly suppressed the MPP-induced apoptosis and fibrosis. In Masson trichrome staining group, the collagen fibers were stained in blue. In TUNEL staining group, the nucleus of apoptosis cell was stained in brown. 
2.5. Transmission Electron Microscopy. To examine the formation of autophagosomes in lung tissue, transmission electron microscopy was performed as previously described [16]. Briefly, lung tissue $\left(1 \mathrm{~mm}^{3}\right)$ from each group was fixed with ice-cold glutaraldehyde $(2.5 \%$ in $0.1 \mathrm{M}$ cacodylate buffer, $\mathrm{pH}$ 7.4) for $1 \mathrm{~h}$, postfixed in $\mathrm{OsO}_{4}$, and embedded in epoxy resin. Ultrathin sections $(70-80 \mathrm{~nm})$ were stained with uranyl acetate and lead citrate and examined in a CM-120 electron microscopy (Philips, Holland).

2.6. Enzyme-Linked Immunosorbent Assay. TGF- $\beta$, IL-6, IL$1 \beta$, and TNF- $\alpha$ in the supernatants of BEAS-2B or serum from subjects with MPP or healthy volunteers were measured using human ELISA kits (Sen-Xiong Company, Guangdong, China); TGF- $\beta$, IL-6, IL- $1 \beta$, and TNF- $\alpha$ in serum from mice with different treatment were measured using commercially available mice ELISA kits (Sen-Xiong Company, Guangdong, China). In accordance with manufacturer's instructions, supernatants were stored at $-80^{\circ} \mathrm{C}$ before measurement and both standards and samples were run in triplicate. The $\mathrm{OD}_{450}$ was calculated and standard curves were plotted.

2.7. Western Blotting Analysis. Western blotting was performed using lung tissue homogenates in urea buffer ( $8 \mathrm{M}$ urea, $1 \mathrm{M}$ thiourea, $0.5 \%$ CHAPS, $50 \mathrm{mM}$ dithiothreitol, and $24 \mathrm{mM}$ spermine). Protein fractions were prepared using a protein extraction kit (Pierce, Waltham, MA, USA) following manufacturer's protocols. GAPDH was used as a loading control. Samples ( $40 \mu \mathrm{g}$ total protein) were separated with SDS-PAGE and transferred to nitrocellulose membranes (Millipore, Billerica, MA, USA). After blocking in 5\% nonfat milk for $1 \mathrm{~h}$, membranes were incubated with primary antibodies against $\alpha$-SMA, collagen I, collagen III, Beclin-1, LC-3, P62, TGF- $\beta$, and GAPDH at $4^{\circ} \mathrm{C}$ overnight. After washing, membranes were incubated with horseradish peroxidase-conjugated secondary antibodies for $1 \mathrm{~h}$ at room temperature. Signals were detected using an ECL detection system (GE Healthcare, Chicago, IL, USA) and analyzed using ImageJ 1.42q software (National Institute of Health, Bethesda, USA). The in vitro experiment was independent repeated three times. The in vivo test was repeated three times in each animal.

2.8. Measurement of Cell Viability via MTT Assay. For the determination of cell viability, BEAS-2B cells were treated with NRG $(0,25,50,100$, and $250 \mu \mathrm{M})$ for $24 \mathrm{~h}$ and then cultured with MTT solution $(5 \mathrm{mg} / \mathrm{ml})$ (Sigma, St. Louis, MO, USA) for $4 \mathrm{~h}$. The viable cells converted MTT to formazan, which generated a blue purple color after dissolving in $150 \mu \mathrm{l}$ of dimethyl sulfoxide. The absorbance at $590 \mathrm{~nm}$ was measured using a Multiskan ${ }^{\mathrm{TM}}$ GO microplate spectrophotometer (Thermo Fisher Scientific, Inc., Cleveland, USA). All assays were repeated as independent experiments at least twice.

2.9. Flow Cytometric Determination of Cell Death. Cell death was analyzed using an annexin $\mathrm{V}$ apoptosis detection kit (eBioscience, California, USA) as previously described [17]. Briefly, both floating and adherent BEAS-2B cells were collected and resuspended in $100 \mu \mathrm{l}$ of annexin V-binding buffer. After staining with $5 \mu$ l of FITC-conjugated annexin $\mathrm{V}$ for $15 \mathrm{~min}$ at room temperature in the dark, the cells were washed and incubated with propidium iodide (PI). All cell samples were assayed using a FACSAria flow cytometer (BD Biosciences, New Jersey, USA), and the acquired data were further analyzed using FCS Express (De Novo Software, California, USA).

2.10. Autophagy Assay. Autophagy was determined by detection of the processing of the autophagy marker LC3 and fluorescence microscopic detection of the formation of the autophagosomes in cells transfected with GFP-LC3.

2.11. Immunohistochemistry Assay. Lung tissue samples were fixed in $10 \%$ formalin solution and embedded in paraffin. $5 \mu \mathrm{m}$ sections were stained with TUNEL and Masson's trichrome. Sections were examined using an Axiophot light microscope (Zeiss, Oberkochen, Germany) and photographed with a digital camera.

2.12. Statistical Analysis. Results are expressed as the mean \pm standard deviation (SD). Statistical significance was evaluated by analysis of variance followed by Tukey-Kramer multiple comparison test and by Student's $t$-test. $P<0.05$ denotes statistical significance.

\section{Results}

3.1. Comparison of Clinical and Laboratory Characteristics between Children with and without MPP. The characteristics of the subjects are shown in Table 1. There was no difference with respect to the mean age and sex among the two groups. The results show that children with MP pneumonia have a higher ratio of neutrophils and white blood cells in whole blood cell analysis. There was no significant difference in lymphocytes and blood platelets between the two groups. This suggests that MP infection can induce the inflammatory response.

The serum cytokine levels (IL-6, IL- $1 \beta$, and TNF- $\alpha$ ) were significantly higher in children with MP pneumonia than in those with nonatopic MP pneumonia $(P<0.05)$. The serum TGF- $\beta$ levels were also significantly higher in children with MP pneumonia, suggesting that MP infection can induce pulmonary fibrosis because TGF- $\beta$ plays an important role in mediating pulmonary fibrosis $[18,19]$.

3.2. NRG Treatment Decreased M. pneumonia-Induced Lung Injury in Mice by Inhibiting Inflammatory Cytokine Expression and Pulmonary Fibrosis. As shown in Figure 1, in M. pneumonia challenged mice, serum IL-6 (Figure 1(b)), IL-1 $\beta$ (Figure 1(c)), and TNF- $\alpha$ (Figure 1(d)) were significantly higher in mice after 1 month infection than their saline controls. NRG treatment decreased MPinduced inflammatory cytokine expression after MP infection in mice serum. ELISA has shown that NRG treatment suppressed MP-induced TGF- $\beta$ expression (Figure 1(e)) suggesting that NRG can inhibit MP-induced fibrosis as has been previously reported [20]. Immunohistochemistry has shown that NRG treatment suppressed MP-induced apoptosis (Figure 1(f)) and pulmonary fibrosis (Figure 1(g)). 

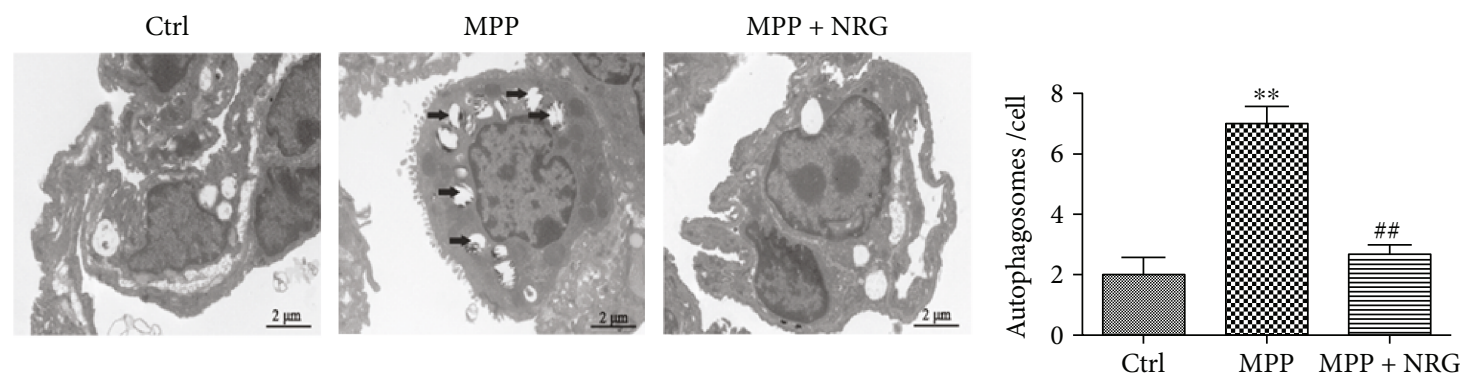

(a)

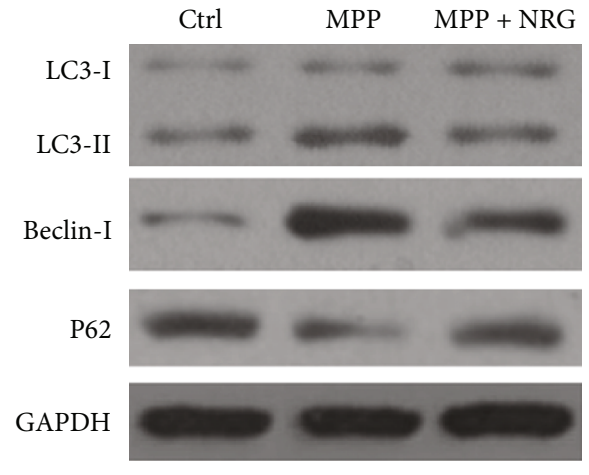

(b)

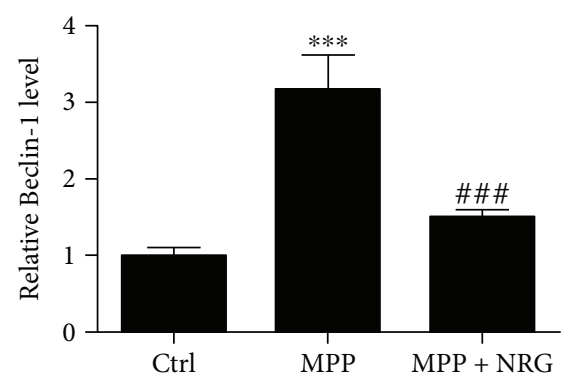

(d)

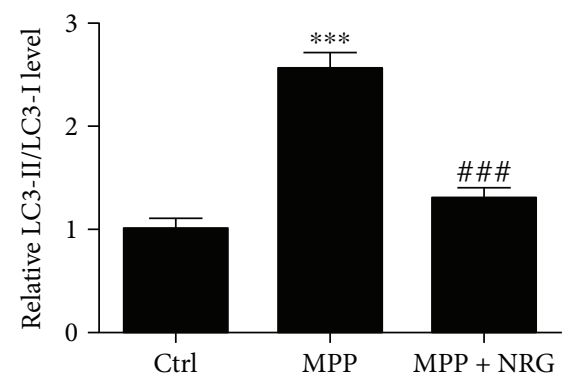

(c)

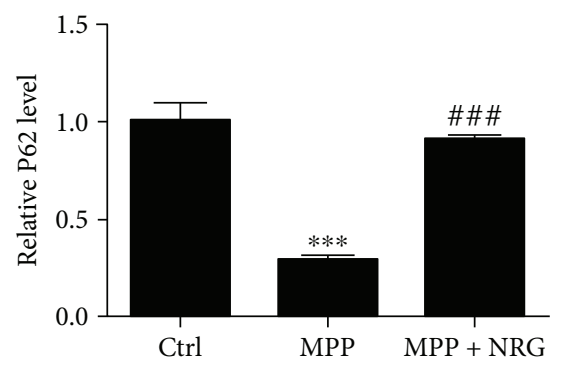

(e)

Figure 2: NRG treatment decreased $M$. pneumonia-induced autophagy. (a) TEM shows the autophagosome in lung tissues. Data are presented as the mean \pm SD with Tukey-Kramer multiple comparison test analysis. $n=10{ }^{* *} P<0.01$ versus ctrl group; ${ }^{\# \#} P<0.01$ versus MPP group. (b) Western blot detection shows the expression of autophagy relative protein LC3, P62, and Beclin-1. (c-e) The results are expressed as relative ratios of band density of LC3-II/LC3-I (c), Beclin-1 (d), and P62 (e). Data are presented as the mean \pm SD with Tukey-Kramer multiple comparison test analysis. $n=3 .{ }^{* *} P<0.001$ versus ctrl group; ${ }^{\# \#} P<0.001$ versus MPP group.

3.3. NRG Treatment Decreased M. pneumonia-Induced Autophagy. In order to identify whether MPP can induce autophagy and to verify the protective effect of NRG on MPP-induced lung inflammation and fibrosis relative to autophagy after MP infection and treatment with NRG for 1 month, lung tissues were collected for transmission electron microscope (TEM) analysis. The results of TEM show that the autophagosomes in lung tissues were increased after MP infection and that NRG treatment inversed the progress (Figure 2(a)). Western blot detection shows the expression of autophagy relative protein LC3, P62, and Beclin-1 (Figures 2(b)-2(e)). The results demonstrate that NRG suppressed MP-induced autophagy relative protein LC3 and Beclin-1 expression and increased MP-induced P62 inhibition. The conversion of LC3-I into LC3-II is an essential step in autophagosome formation, and the abundance of LC3-II correlates with the number of autophagosomes, suggesting a protective effect of NRG on MP-induced inflammation and fibrosis relative to autophagy regulation.

\subsection{NRG Treatment Inhibits MP-Induced BEAS-2B Cells} Injury by Inhibiting Autophagy. To confirm the relationship between autophagy, inflammation, and fibrosis, BEAS-2B cells were employed. MTT analysis shows that NRG treatment has no significantly effect on cell viability with different concentrations $(0,25,50,100$, and $500 \mu \mathrm{M})$, suggesting that NRG has no cytotoxicity (Figure 3(a)). FITC-annexin V/PI staining and flow cytometry analysis show that the apoptosis ratio of BEAS-2B cells was increased after MP 


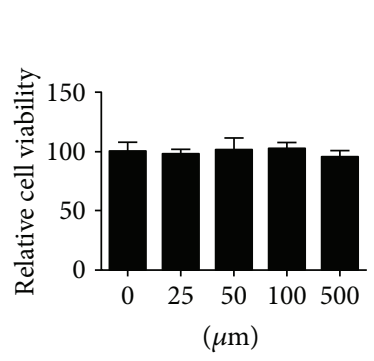

(a)

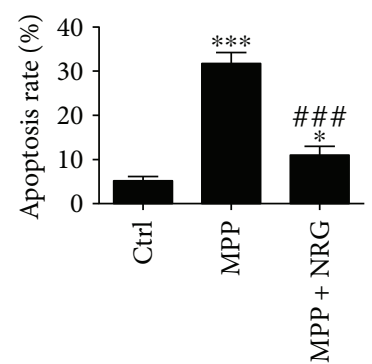

(c)

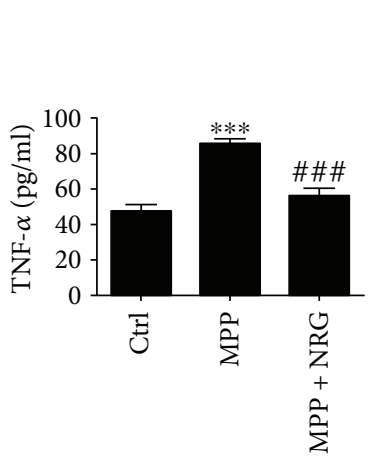

(f)

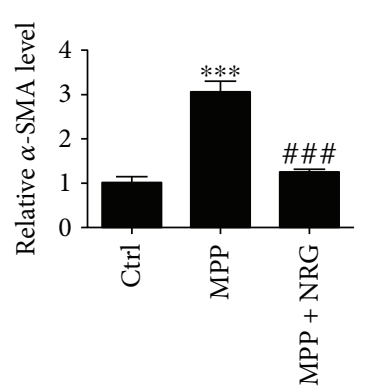

(i)
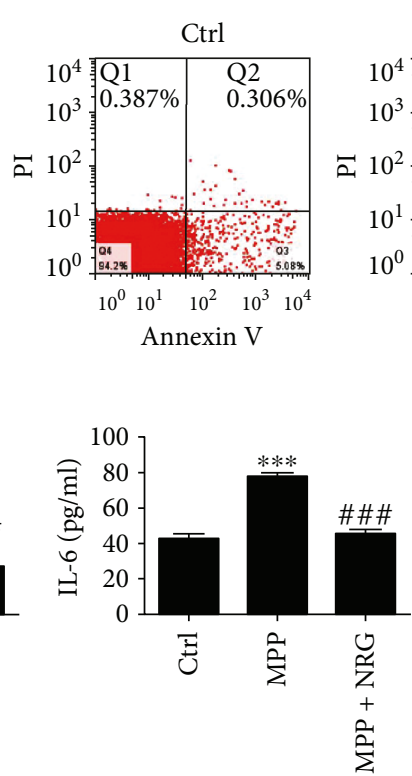

(d)

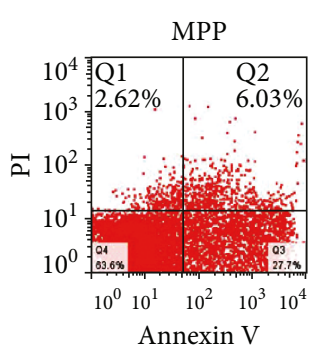

(b)
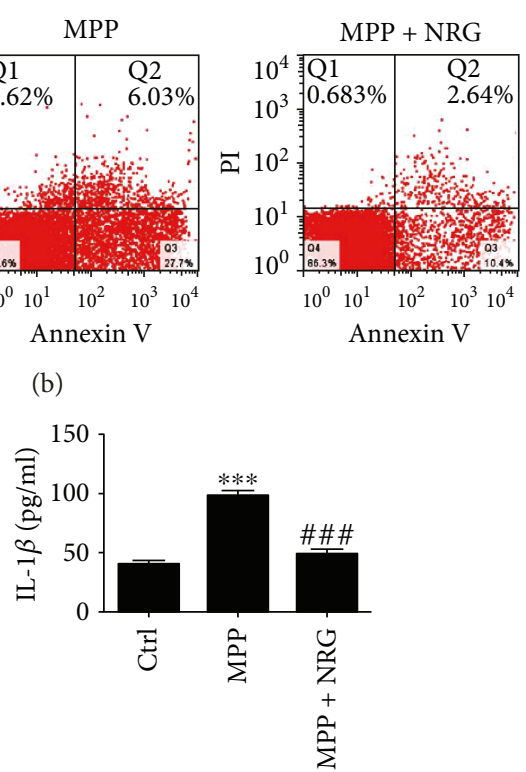

(e)

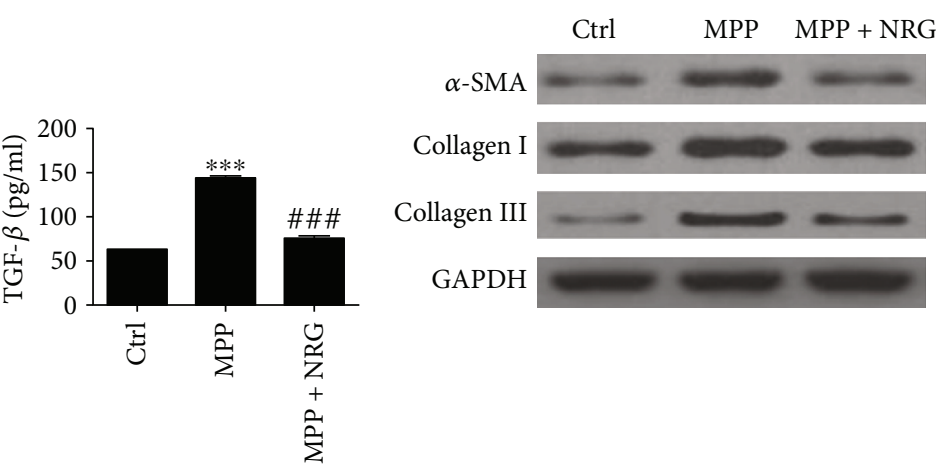

(g)

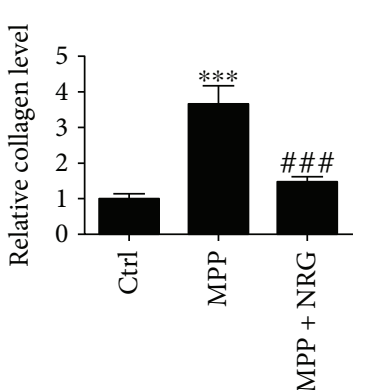

(j)

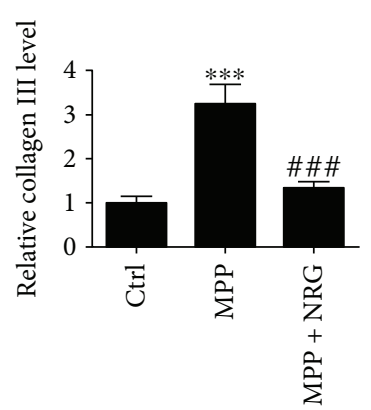

(k)

FIGURE 3: NRG treatment inhibits MP-induced BEAS-2B cells injury. (a) MTT analysis shows that NRG treatment has no significant effect on cell viability with different concentrations $(P>0.05)$. ( $\mathrm{b}$ and $\mathrm{c}$ ) Apoptosis of BEAS-2B cells was measured with FITC-annexin V/PI staining and flow cytometry in response to different treatments. Data are presented as the mean \pm SD with Tukey-Kramer multiple comparison test analysis. $n=3$. ${ }^{*} P<0.05,{ }^{* * *} P<0.001$ versus ctrl group; ${ }^{\# \# \# ~} P<0.001$ versus MPP group. (d-f) Inflammatory cytokines IL-6, IL- $1 \beta$, and TNF- $\alpha$ expression in cell supernatant were measured with ELISA. (g) TGF- $\beta$ expression in cell supernatant was measured with ELISA. Data are presented as the mean \pm SD with Tukey-Kramer multiple comparison test analysis. $n=5$. ${ }^{* * *} P<0.001$ versus ctrl group; \#\#\# $P<0.001$ versus MPP group. (h-k) Fibrotic correlation factor $\alpha$-SMA, collagen I, and collagen III expression in cell were measured with western blot. Data are presented as the mean $\pm \mathrm{SD}$ with Tukey-Kramer multiple comparison test analysis. $n=3 .{ }^{* * *} P<0.001$ versus

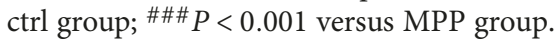

infection for $24 \mathrm{~h}$. NRG treatment $(100 \mu \mathrm{M})$ reversed MPinduced BEAS-2B cells apoptosis (Figures 3(b) and 3(c)). Inflammatory cytokines IL-6, IL- $\beta, \quad \mathrm{TNF}-\alpha$, and transforming growth factor beta (TGF- $\beta$ ) expression in cell supernatant were measured with ELISA (Figures 3(d)-3(g)). The results show that NRG can significantly inhibit MP- 
Ctrl
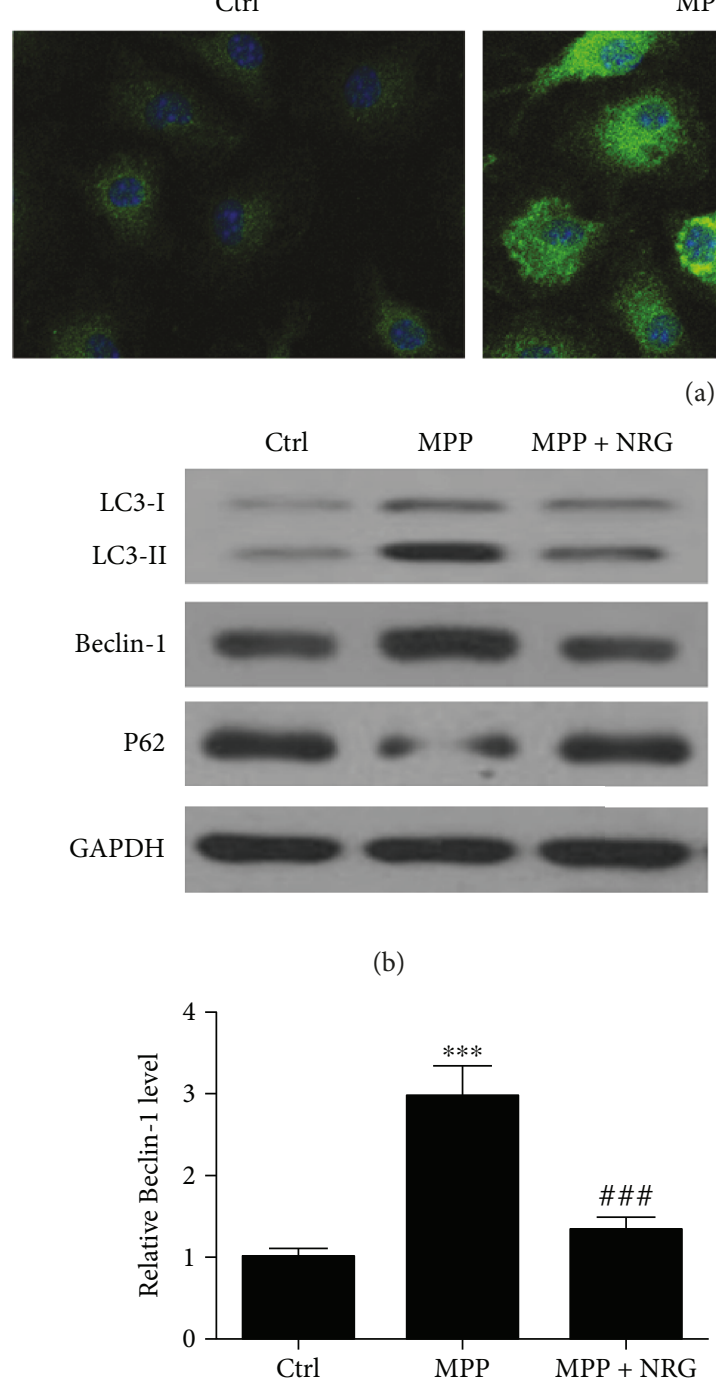

(d)
P62

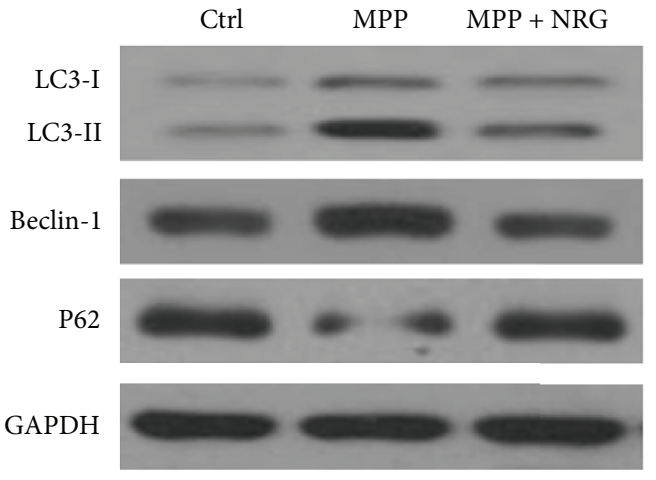

GAPDH

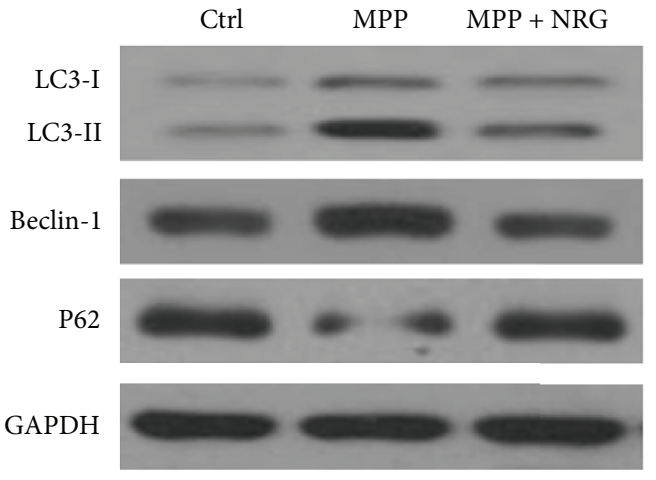

(b)
MPP

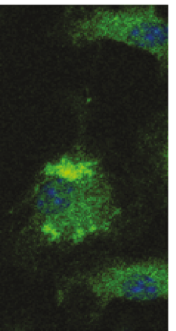

a)

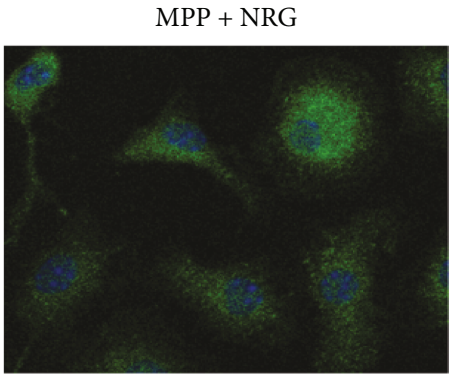

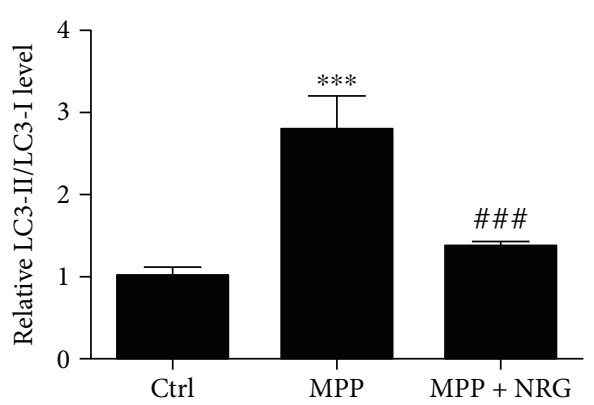

(c)

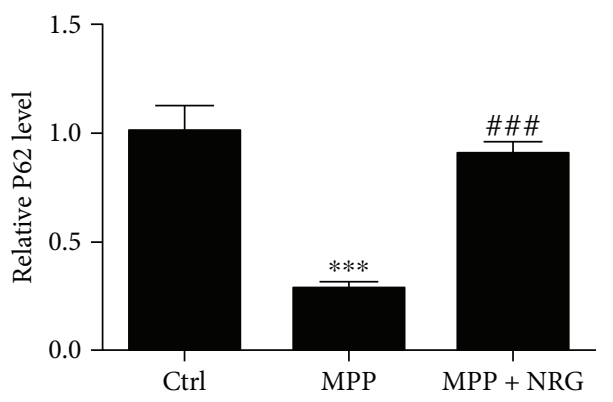

(e)

FIGURE 4: NRG treatment inhibits M. pneumonia-induced autophagy. (a) Immunofluorescence shows the effect of NRG treatment on MPinduced autophagy plaque production. ( $\mathrm{b}-\mathrm{e}$ ) Western blot detection shows the expression of autophagy relative protein LC3, P62, and Beclin1 expression. Data are presented as the mean $\pm \mathrm{SD}$ with Tukey-Kramer multiple comparison test analysis. $n=3$. ${ }^{* * *} P<0.001$ versus ctrl group; \#\# $P<0.001$ versus MPP group.

induced inflammatory cytokines and fibrin-related protein TGF- $\beta$ expression. The results are consistent with previous clinical and animal experiments. Western blot confirmed that fibrotic correlation factor $\alpha$-SMA, collagen I, and collagen III expression in BEAS-2B cells were decreased with NRG treatment.

To identify the effect of NRG on MP-induced autophagy, BEAS-2B cells were transfected with LC3-GFP overexpression vector prior to MP infection $(20 \mathrm{ng} / \mathrm{ml})$ and NRG treatment. Immunofluorescence shows that NRG treatment reversed MP-induced autophagy plaque production (Figure 4(a)). Western blot detection confirmed that NRG inhibits MP-induced autophagy relative protein LC3 and Beclin-1 expression, but increased P62 expression (Figures 4(b)-4(e)).
3.5. Autophagy Inhibitor 3-Ma Treatment Inhibits $M$. pneumonia-Induced BEAS-2B Cell Injury. To further confirm the relationship between autophagy, inflammation, and fibrosis, BEAS-2B cells treated with 3-Ma $(4 \mathrm{mM})$ for $2 \mathrm{~h}$ prior to treatment with $20 \mathrm{ng} / \mathrm{ml} \mathrm{MP}$ for up to $24 \mathrm{~h}$. FITCannexin V/PI staining and flow cytometry analysis show that MP-induced apoptosis of BEAS-2B cells was reduced with autophagy inhibition (Figures 5(a) and 5(b)). Inflammatory cytokines IL-6, IL-1 $\beta$, and TNF- $\alpha$ expression in cell supernatant were measured by ELISA. The results show that autophagy inhibition reversed MP-induced inflammatory cytokine expression (Figures 5(c)-5(e)). ELISA and western blot analysis also show that autophagy inhibition reversed MP-induced fibrosis-related protein expression (Figures 5(f)-5(j)). In conclusion, we found NRG inhibits 


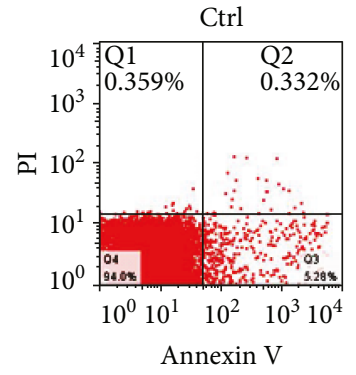

Annexin V

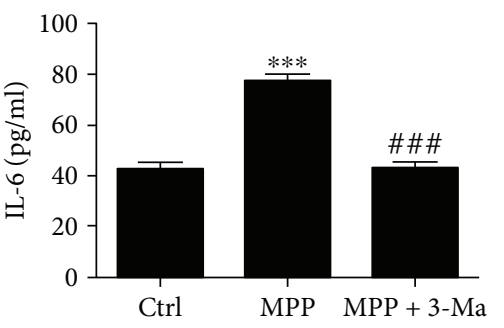

(c)

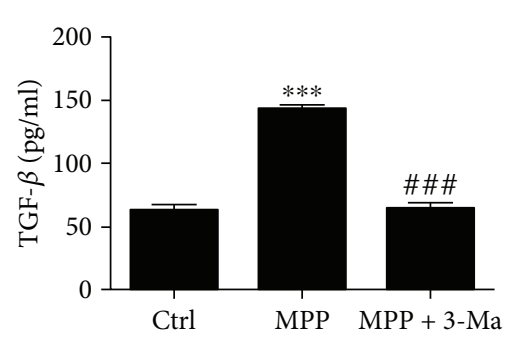

(f)

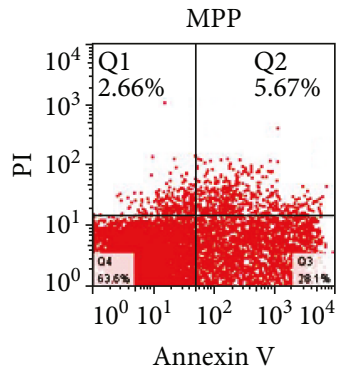

(a)

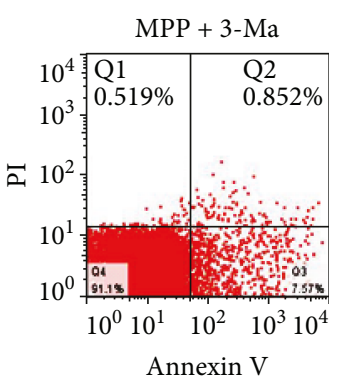

Annexin V

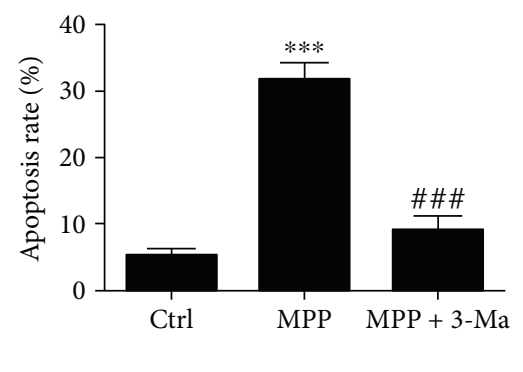

(b)

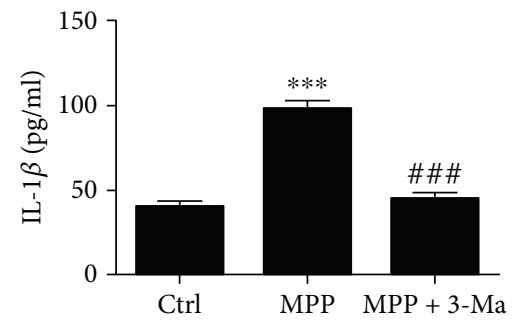

(d)

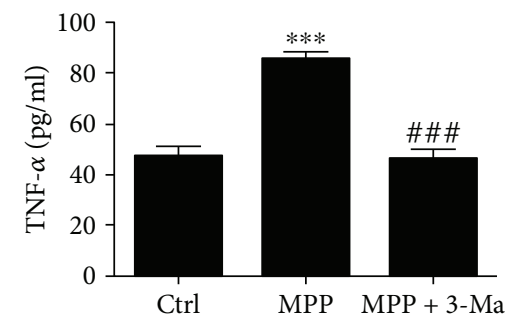

(e)

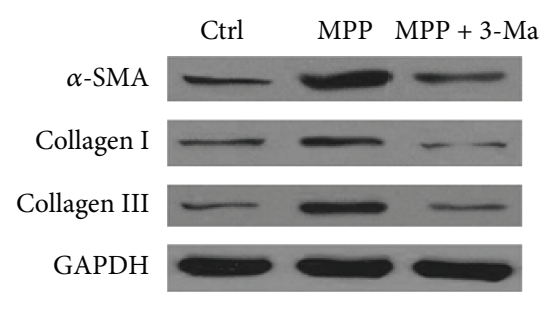

(g)

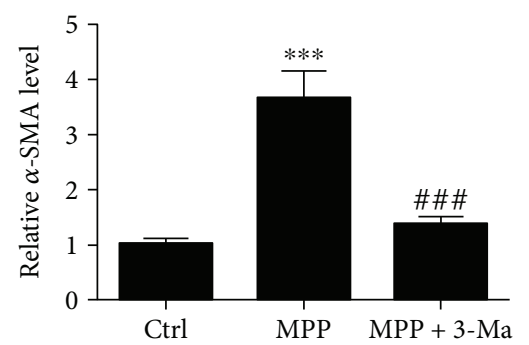

(h)

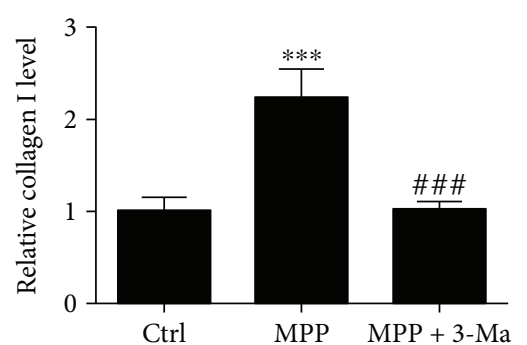

(i)

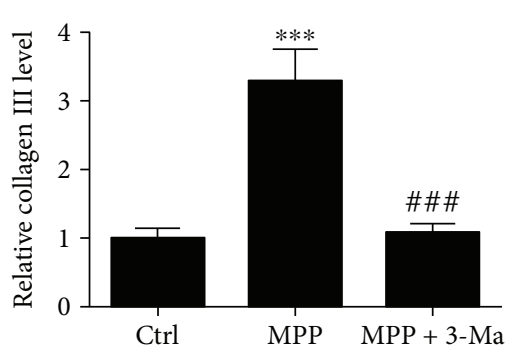

(j)

Figure 5: Autophagy inhibitor 3-Ma treatment inhibits M. pneumonia-induced BEAS-2B cells injury. BEAS-2B cells treated with 3-Ma $(4 \mathrm{mM})$ for $2 \mathrm{~h}$ prior stimulated with $20 \mathrm{ng} / \mathrm{ml} \mathrm{MP}$ for up to $24 \mathrm{~h}$. (a and b) Apoptosis of BEAS-2B cells was measured with FITCannexin V/PI staining and flow cytometry. Data are presented as the mean \pm SD with Tukey-Kramer multiple comparison test analysis. $n=3$. ${ }^{* * *} P<0.001$ versus ctrl group; ${ }^{* \#} P<0.001$ versus MPP group. (c-e) Inflammatory cytokines IL-6, IL- $1 \beta$, and TNF- $\alpha$ expression in cell supernatant were measured by ELISA. Data are presented as the mean \pm SD with Tukey-Kramer multiple comparison

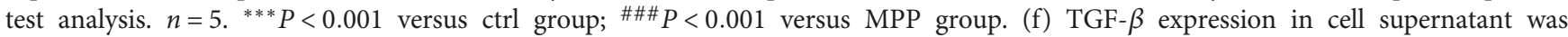
measured with ELISA. Data are presented as the mean \pm SD with Tukey-Kramer multiple comparison test analysis. $n=5$. ${ }^{* * *} P<0.001$ versus ctrl group; \#\#\# $P<0.001$ versus MPP group. (g-j) Fibrotic correlation factor $\alpha$-SMA, collagen I, and collagen III expression in cell were measured with western blot. $n=3 .{ }^{* * *} P<0.001$ versus ctrl group; ${ }^{\# \# \# ~} P<0.001$ versus MPP group.

MP-induced inflammation and pulmonary fibrosis by inhibiting autophagy.

\section{Discussion}

Mycoplasma pneumoniae causes primary atypical pneumonia, tracheobronchitis, pharyngitis, and asthma in humans
$[21,22]$. Moreover, $3 \%-64 \%$ of pediatric asthma patients in the chronic stable phase are infected with $M$. pneumoniae. Thus, MP is a known aggravating factor of asthmatic symptoms in both acute exacerbations and the chronic stable phase. This is further demonstrated by the alleviation of respiratory system symptoms and improvement of pulmonary function after use of macrolides to treat MP infection 
[23]. The symptoms of pneumonia caused by $M$. pneumoniae are correlated with the induction of proinflammatory cytokines [24] and induced pulmonary fibrosis [25], suggesting that the excessive immune responses induced by M. pneumoniae play an important role in the development of pneumonia.

The involvement of autophagy in the pathogenesis of asthma was demonstrated previously by microscopic examination showing autophagosome formation in fibroblasts from asthmatic patients [26]. Furthermore, increased airway responsiveness and inflammatory cytokine expression were associated with LC3 upregulation and increased autophagosome formation in eosinophils in an ovalbumin-induced asthma mouse model, suggesting the involvement of autophagy in allergic inflammation [27]. Increasing evidence shows that inhibiting autophagosome formation in airways can significantly suppress bronchial fibrosis and the inflammatory response $[28,29]$. So, the modulation of autophagy may have the potential to treat common respiratory ailments and disorders.

Our previous study has shown that the Chinese medicine, QTF, has been used for the clinical treatment of MPP and with a significantly therapeutic effect. NRG, an important component of QTF, has been reported to play an important in anti-inflammatory and antifibrotic role $[10,30]$. This study aimed to clarify the effect of NRG on MP-induced inflammatory and lung fibrosis in pneumonia. To investigate the relationship between autophagy, inflammation, and fibrosis, our study found that inflammatory and fibrosisrelated protein expression was significantly increased in both clinical, in vitro, and in vivo experiments after MP infection. The results of in vitro and in vivo experiments also show that MP infection promotes autophagy. NRG treatment reversed MP-induced lung inflammation and fibrosis by inhibiting autophagy. The results were confirmed by 3-Ma treatment (an autophagy inhibitor). Based on the data presented, we have shown the protective effect of NRG on airway remodeling after MP infection by inhibiting autophagy-mediated lung inflammation and fibrosis.

\section{Disclosure}

Zhen Xiao and Zhiyan Jiang are cocorresponding authors.

\section{Conflicts of Interest}

The authors declare no competing interests.

\section{Authors' Contributions}

Zhen Xiao and Zhiyan Jiang participated in performing the experiments and writing of the paper; they contributed equally to the work. Yan Lin and Dan Tan participated in the data analysis. Qianna Kan and Dan Tan provided substantial advice in designing the study, assisting in the division of labor, and revising the paper; they contributed equally to the work.

\section{Acknowledgments}

This work was supported by the National Natural Science Foundation of China Project (Grant nos. 81574021 and 81674024).

\section{References}

[1] H. Zhao, S. Li, L. Cao et al., "Surveillance of Mycoplasma pneumoniae infection among children in Beijing from 2007 to 2012," Chinese Medical Journal, vol. 127, no. 7, pp. 12441248, 2014.

[2] T. P. Atkinson, M. F. Balish, and K. B. Waites, "Epidemiology, clinical manifestations, pathogenesis and laboratory detection of Mycoplasma pneumoniae infections," FEMS Microbiology Reviews, vol. 32, no. 6, pp. 956-973, 2008.

[3] T. Shimizu, "Inflammation-inducing factors of Mycoplasma pneumoniae," Frontiers in Microbiology, vol. 7, 2016.

[4] T. Shimizu, "Pathogenic factors of mycoplasma," Nihon Saikingaku Zasshi, vol. 70, no. 4, pp. 369-374, 2015.

[5] O. C. Tablan and M. P. Reyes, "Chronic interstitial pulmonary fibrosis following Mycoplasma pneumoniae pneumonia," The American Journal of Medicine, vol. 79, no. 2, pp. 268270, 1985.

[6] N. Mizushima, "Autophagy: process and function," Genes \& Development, vol. 21, no. 22, pp. 2861-2873, 2007.

[7] A. Orvedahl and B. Levine, "Eating the enemy within: autophagy in infectious diseases," Cell Death and Differentiation, vol. 16, no. 1, pp. 57-69, 2008.

[8] H. W. Virgin and B. Levine, "Autophagy genes in immunity," Nature Immunology, vol. 10, no. 5, pp. 461-470, 2009.

[9] T. Shimizu, Y. Kimura, Y. Kida et al., "Cytadherence of Mycoplasma pneumoniae induces inflammatory responses through autophagy and toll-like receptor 4," Infection and Immunity, vol. 82, no. 7, pp. 3076-3086, 2014.

[10] E. Hernandez-Aquino, N. Zarco, S. Casas-Grajales et al., "Naringenin prevents experimental liver fibrosis by blocking TGF $\beta$-Smad3 and JNK-Smad3 pathways," World Journal of Gastroenterology, vol. 23, no. 24, pp. 4354-4368, 2017.

[11] F. Z. Hua, J. Ying, J. Zhang et al., "Naringenin pre-treatment inhibits neuroapoptosis and ameliorates cognitive impairment in rats exposed to isoflurane anesthesia by regulating the PI3/ Akt/PTEN signalling pathway and suppressing NF- $\kappa$ B-mediated inflammation," International Journal of Molecular Medicine, vol. 38, no. 4, pp. 1271-1280, 2016.

[12] Y. Zhao, D. Fan, B. Ru et al., "6-C-(E-phenylethenyl)naringenin induces cell growth inhibition and cytoprotective autophagy in colon cancer cells," European Journal of Cancer, vol. 68, pp. 38-50, 2016.

[13] R. Ali, A. Shahid, N. Ali, S. K. Hasan, F. Majed, and S. Sultana, "Amelioration of benzo[a]pyrene-induced oxidative stress and pulmonary toxicity by naringenin in Wistar rats: a plausible role of COX-2 and NF- $\kappa \mathrm{B}$," Human \& Experimental Toxicology, vol. 36, no. 4, pp. 349-364, 2016.

[14] A. Orvedahl, S. MacPherson, R. Sumpter Jr, Z. Talloczy, Z. Zou, and B. Levine, "Autophagy protects against Sindbis virus infection of the central nervous system," Cell Host \& Microbe, vol. 7, no. 2, pp. 115-127, 2010.

[15] H. W. Chu, J. G. Rino, R. B. Wexler, K. Campbell, R. J. Harbeck, and R. J. Martin, "Mycoplasma pneumoniae infection increases airway collagen deposition in a murine model 
of allergic airway inflammation," American Journal of Physiology. Lung Cellular and Molecular Physiology, vol. 289, no. 1, pp. L125-L133, 2005.

[16] C. Luo, M. W. Ouyang, Y. Y. Fang et al., "Dexmedetomidine protects mouse brain from ischemia-reperfusion injury via inhibiting neuronal autophagy through up-regulating HIF-1 $\alpha$, Frontiers in Cellular Neuroscience, vol. 11, p. 197, 2017.

[17] J. Tao, H. Wang, Y. Zhai et al., "Downregulation of Nrf2 promotes autophagy-dependent osteoblastic differentiation of adipose-derived mesenchymal stem cells," Experimental Cell Research, vol. 349, no. 2, pp. 221-229, 2016.

[18] X. H. Chang, A. Zhu, F. F. Liu et al., "Nickel oxide nanoparticles induced pulmonary fibrosis via TGF- $\beta 1$ activation in rats," Human \& Experimental Toxicology, vol. 36, no. 8, pp. 802-812, 2017.

[19] A. M. Kabel, M. S. Omar, and M. A. A. Elmaaboud, "Amelioration of bleomycin-induced lung fibrosis in rats by valproic acid and butyrate: role of nuclear factor kappa-B, proinflammatory cytokines and oxidative stress," International Immunopharmacology, vol. 39, pp. 335-342, 2016.

[20] A. L. Tatler, A. T. Goodwin, O. Gbolahan et al., "Amplification of TGF $\beta$ induced ITGB6 gene transcription may promote pulmonary fibrosis," PLoS One, vol. 11, no. 8, article e0158047, 2016.

[21] J. C. Gil, R. L. Cedillo, B. G. Mayagoitia, and M. D. Paz, "Isolation of Mycoplasma pneumoniae from asthmatic patients," Annals of Allergy, vol. 70, no. 1, pp. 23-25, 1993.

[22] K. B. Waites and D. F. Talkington, "Mycoplasma pneumoniae and its role as a human pathogen," Clinical Microbiology Reviews, vol. 17, no. 4, pp. 697-728, 2004.

[23] V. Hernando-Sastre, "Macrolide antibiotics in the treatment of asthma. An update," Allergologia et Immunopathologia, vol. 38, no. 2, pp. 92-98, 2010.

[24] J. K. Larsen, “Chapter 23 Washless double staining of a nuclear antigen (Ki-67 or bromodeoxyuridine) and DNA in unfixed nuclei," Methods in Cell Biology, vol. 33, pp. 227-234, 1990.

[25] D. I. Sanchez, M. P. Perez-Canto, M. G. Urcelay, B. C. Garcia, L. E. Vega-Briceno, and B. S. Gonzalez, "Progressive respiratory insufficiency secondary to pulmonary fibrosis in childhood. Report of one case," Revista Médica de Chile, vol. 133, no. 1, pp. 82-88, 2005.

[26] Y. C. Lee, S. Jogie-Brahim, D. Y. Lee et al., "Insulin-like growth factor-binding protein-3 (IGFBP-3) blocks the effects of asthma by negatively regulating NF- $\kappa \mathrm{B}$ signaling through IGFBP-3R-mediated activation of caspases," Journal of Biological Chemistry, vol. 286, no. 20, pp. 17898-17909, 2011.

[27] J. N. Liu, D. H. Suh, H. K. Trinh, Y. J. Chwae, H. S. Park, and Y. S. Shin, "The role of autophagy in allergic inflammation: a new target for severe asthma," Experimental \& Molecular Medicine, vol. 48, no. 7, article e243, 2016.

[28] I. H. Cho, Y. J. Choi, J. H. Gong, D. Shin, M. K. Kang, and Y. H. Kang, "Astragalin inhibits autophagy-associated airway epithelial fibrosis," Respiratory Research, vol. 16, no. 1, p. 51, 2015.
[29] S. M. Casalino-Matsuda, A. Nair, G. J. Beitel, K. L. Gates, and P. H. Sporn, "Hypercapnia inhibits autophagy and bacterial killing in human macrophages by increasing expression of Bcl-2 and Bcl-xL," The Journal of Immunology, vol. 194, no. 11, pp. 5388-5396, 2015.

[30] J. Liang, Y. Halipu, F. Hu et al., "Naringenin protects keratinocytes from oxidative stress injury via inhibition of the NOD2mediated NF- $\kappa$ B pathway in pemphigus vulgaris," Biomedicine \& Pharmacotherapy, vol. 92, pp. 796-801, 2017. 


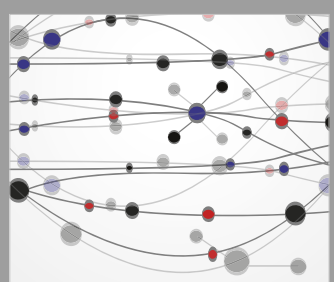

The Scientific World Journal
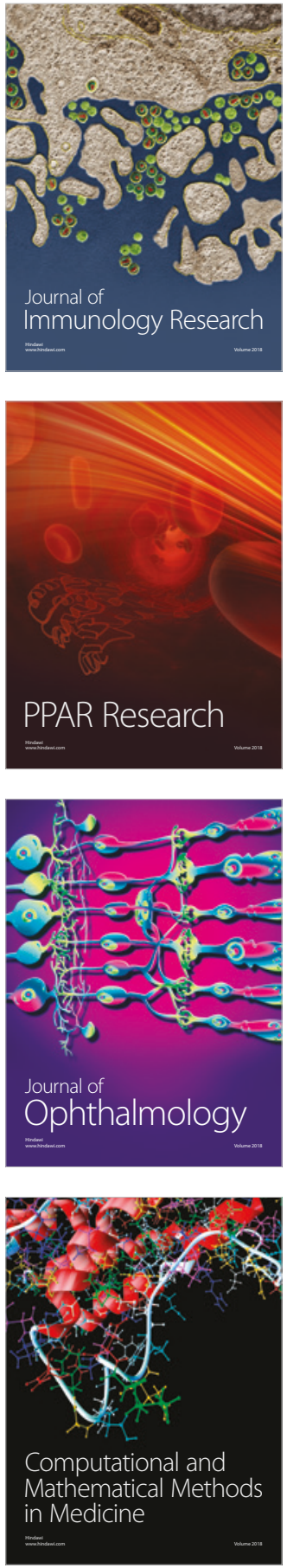

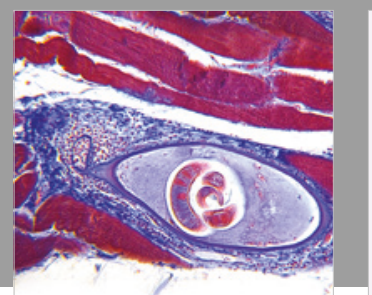

Gastroenterology Research and Practice

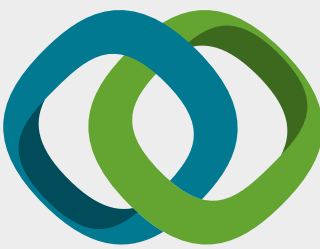

\section{Hindawi}

Submit your manuscripts at

www.hindawi.com
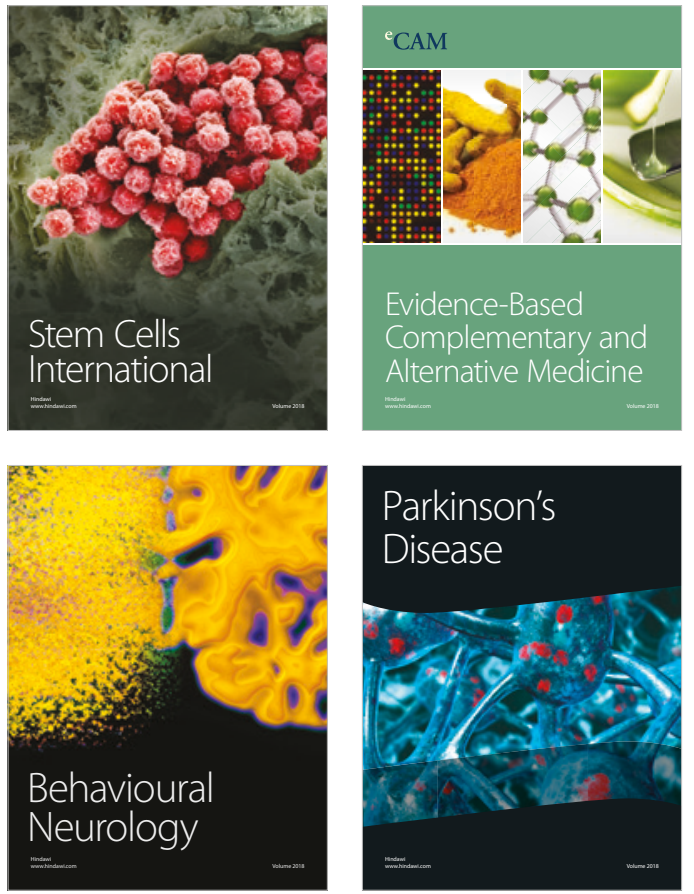

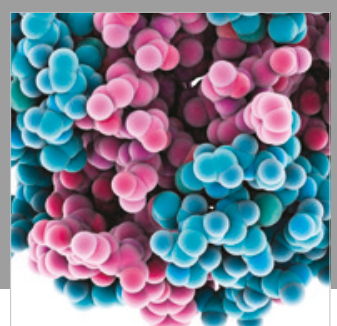

ournal of

Diabetes Research

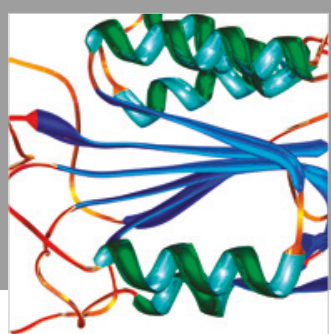

Disease Markers
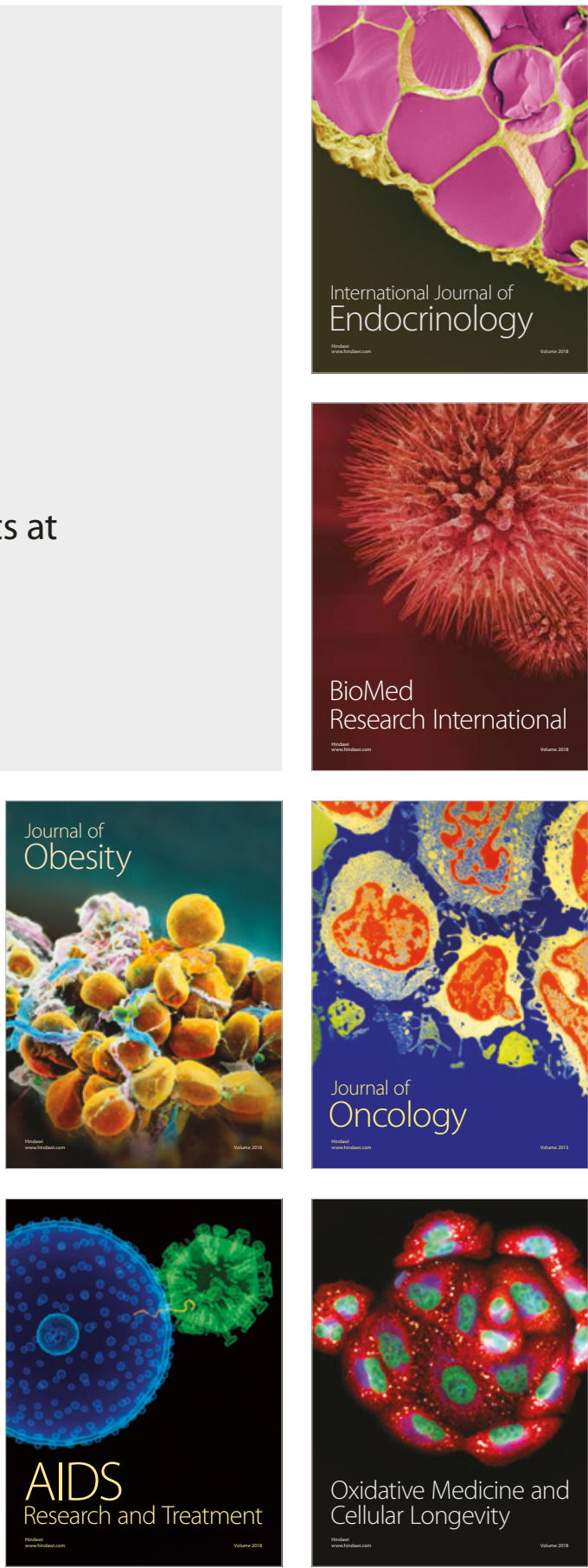